\title{
Ivermectin: a systematic review from antiviral effects to COVID-19 complementary regimen
}

\author{
Fatemeh Heidary $^{1} \cdot$ Reza Gharebaghi ${ }^{2,3}$
}

Received: 21 April 2020 / Revised: 5 May 2020 / Accepted: 17 May 2020 / Published online: 12 June 2020

(c) The Author(s), under exclusive licence to the Japan Antibiotics Research Association 2020

\begin{abstract}
Ivermectin proposes many potentials effects to treat a range of diseases, with its antimicrobial, antiviral, and anti-cancer properties as a wonder drug. It is highly effective against many microorganisms including some viruses. In this comprehensive systematic review, antiviral effects of ivermectin are summarized including in vitro and in vivo studies over the past 50 years. Several studies reported antiviral effects of ivermectin on RNA viruses such as Zika, dengue, yellow fever, West Nile, Hendra, Newcastle, Venezuelan equine encephalitis, chikungunya, Semliki Forest, Sindbis, Avian influenza A, Porcine Reproductive and Respiratory Syndrome, Human immunodeficiency virus type 1 , and severe acute respiratory syndrome coronavirus 2 . Furthermore, there are some studies showing antiviral effects of ivermectin against DNA viruses such as Equine herpes type 1, BK polyomavirus, pseudorabies, porcine circovirus 2 , and bovine herpesvirus 1 . Ivermectin plays a role in several biological mechanisms, therefore it could serve as a potential candidate in the treatment of a wide range of viruses including COVID-19 as well as other types of positive-sense single-stranded RNA viruses. In vivo studies of animal models revealed a broad range of antiviral effects of ivermectin, however, clinical trials are necessary to appraise the potential efficacy of ivermectin in clinical setting.
\end{abstract}

\section{Introduction}

\section{Ivermectin: a multifaced medication}

Ivermectin has been used for several years to treat many infectious diseases in mammals. It has a good safety profile with low adverse effects when orally prescribed. Ivermectin was identified in late 1970s and first approved for animal use in 1981. Its potential use in humans was confirmed a few years later. Subsequently, William C. Campbell and Satoshi Ōmura who discovered and developed this medication received the 2015 Nobel Prize in Physiology or Medicine [1-3].

Fatemeh Heidary

drfatemehheidari@yahoo.com

$\triangle$ Reza Gharebaghi

drgharebaghi@yahoo.com

1 Head of Ophthalmology Division, Taleghani Hospital, Ahvaz Jundishapur University of Medical Sciences, Ahvaz, Iran

2 Kish International Campus, University of Tehran, Tehran, Iran

3 International Virtual Ophthalmic Research Center (IVORC), Austin, TX, USA
Studies revealed that ivermectin as a broad-spectrum drug with high lipid solubility possesses numerous effects on parasites, [1, 3] nematodes, arthropods, flavivirus, mycobacteria, and mammals through a variety of mechanisms. In addition to having antiparasitic and antiviral effects, this drug also causes immunomodulation in the host. Studies have shown its effect on inhibiting the proliferation of cancer cells, as well as regulating glucose and cholesterol in animals. Despite diverse effects of this medication, many of its underlying mechanisms are not yet known [4]. Of note, some of these effects may be secondary to toxic effects on cells (Fig. 1).

\section{COVID-19: a global heath issue}

The severe acute respiratory syndrome coronavirus 2 (SARS-CoV-2) is a single-stranded RNA virus that causes a severe acute respiratory syndrome. The virus was originally called SARS-CoV-2 named officially by World Health Organization as COVID-19 and a global health emergency. The first known case of infection was recorded in early December 2019 and subsequently spread to various continents, including Europe and the United States [5, 6], while the actual behavior of the virus and its pathogenicity are not yet fully understood. 


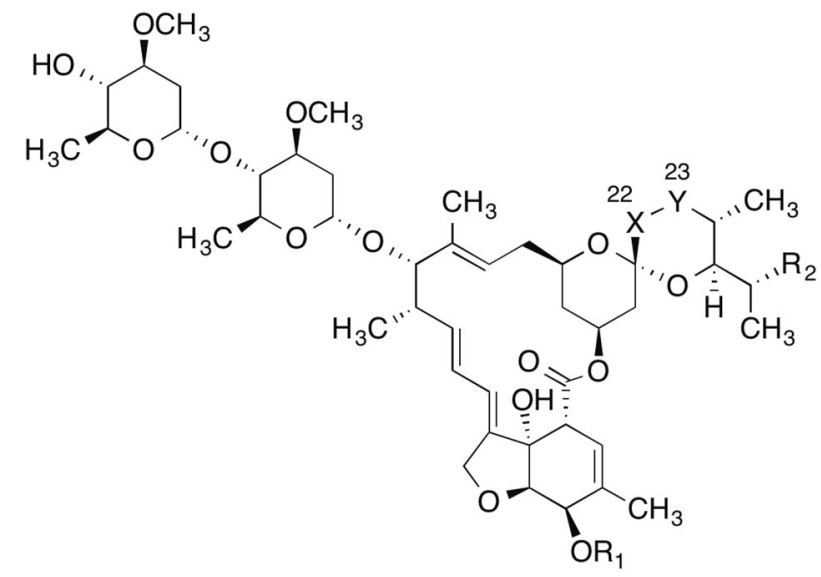

Fig. 1 The molecular structure of avermectin, which then underwent chemical alteration to make ivermectin [45]

Although there has been a history of studies on the virus since few years ago [7, 8], as many types of coronaviruses cause simple respiratory tract infections, but SARS-CoV-1 and MERS caused severe respiratory tract illness in infected humans. The decrease in the threat from SARS and MERS led to decreased research activities against this class of viruses which has led to a lack of preparedness for the new SARS-CoV-2 pandemic.

However, the clinical management guidelines have been revised several times and various antiviral and immunomodulatory agents have been suggested. Herbal medicines in China and other countries have been proposed with doubtful outcomes. Hundreds of clinical trials are currently underway $[9,10]$. On the other hand, several hypotheses have been proposed about the effect of cost-effective and available antibiotics [11, 12], but the effectiveness of those medications has not yet been conclusively proven.

This novel virus has paralyzed not only the world's health care system but also the political and economic relations [13]. As a new chapter in human life opens up [14], the world seems to be divided into two parts pre- and post-COVID-19 era.

Although a few medications have received Emergency Use Authorization for COVID-19 treatment, no proven treatment has been found as yet. A recent in vitro study showed that ivermectin was active against COVID-19infected cell lines [15].

In this study, we summarized the antiviral effects of ivermectin by reviewing available in vivo and in vitro studies over the past 50 years.

\section{Methods}

We conducted a comprehensive search of the PubMed database from January 1, 1970 up to April 14, 2020, using the following syntax constructed using the MeSH Database: (stromectol OR mectizan OR MK-933 OR "MK 933" OR MK933 OR eqvalan OR ivomec OR "bodipy ivermectin" OR (4"-5 AND 7-dimethyl bodipy propionyliverme) OR ivermectin-luminol OR (22 AND 23-dihydroavermectin B1 (a)) OR "dihydroavermectin b1a" OR "h2b1a avermectin" OR "ivermectin component b1a" OR (22 AND 23-Dihydro5-O-demethylavermectin A1a) OR (22 AND 23dihydroavermectin B1a) OR AI3-29390-X OR IVMPO4 OR (22 AND 23-dihydroavermectin B1 (b)) OR (22 AND 23-dihydroavermectin B1b) OR "h2b1b avermectin" OR "ivermectin component b1b" OR (22 AND 23dihydroavermectin B (1) b) OR (avermectin a1a AND 5O-demethyl-25-de (1-methylpropyl)-22,23-dihydro-25-propyl-)) AND (antiviral OR virus OR viral). Articles obtained were reviewed and included when considered appropriate. Also, papers cited in the reference lists of included articles were included when considered appropriate. The retrieved articles were filtered manually to exclude duplicates. There was no language restriction.

\section{Results}

\section{The antiviral effects of ivermectin on RNA viruses}

\section{COVID-19}

In a recent in vitro study, the Vero/hSLAM cells infected with the SARS-CoV-2 or COVID-19 virus were exposed to $5 \mu \mathrm{M}$ ivermectin in $48 \mathrm{~h}$, and a 5000 -fold reduction in viral RNA compared with control was found [15]. The results showed that treatment with ivermectin effectively kills almost all viral particles within $48 \mathrm{~h}$. The study was the first to assess the antiviral effect of ivermectin on COVID-19. The authors acknowledged that the drug may have antiviral effects by inhibiting the importin (IMP) $\alpha / \beta$ receptor, which is responsible for transmitting viral proteins into the host cell nucleus. The authors proposed human studies to confirm the potential benefits of ivermectin in the treatment of COVID-19. Although this study was the first to confirm the antiviral effect of ivermectin on COVID-19 [15], other studies examined the antiviral effects of the drug on both RNA and DNA viruses, which are summarized in Table 1.

\section{Zika virus (ZIKV)}

ZIKV is a single-stranded RNA virus of Flavivirus genus from Flaviviridae family. Barrow et al. in an in vitro study on Zika-infected Huh-7 cells (ZIKMEX_1_7) confirmed the antiviral effect of ivermectin [16]. Ketkar et al. [17] did not find a preventative effect on the Ifnar1 knockout mice 


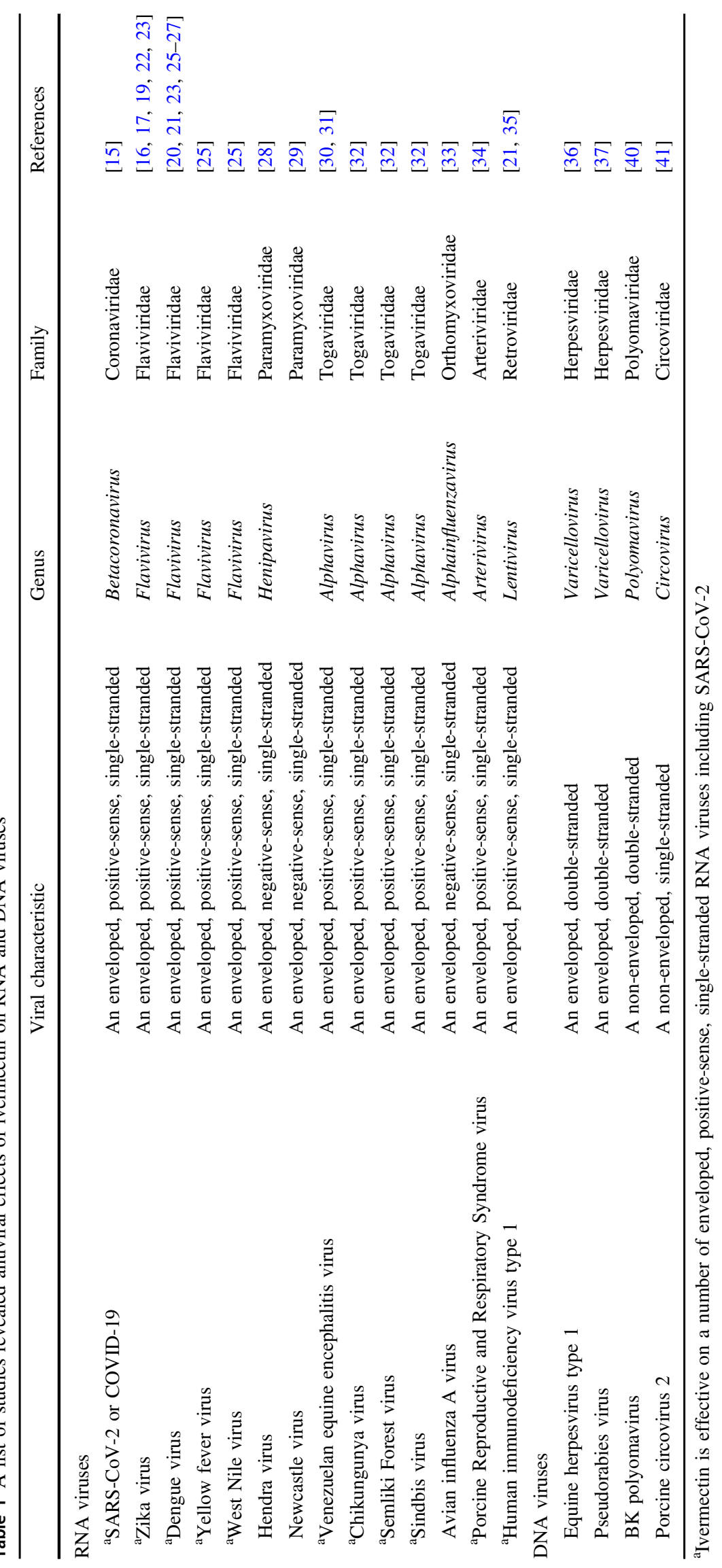


infected with ZIKV treated with $4 \mathrm{mg} \mathrm{kg}^{-1}$ intraperitoneal ivermectin before infection. They also found no difference in mortality and morbidity between the ivermectin-treated and control groups. Results indicate a lack of drug efficacy in this animal model. None of the animals died of druginduced toxicity. Authors justified that low-dose ivermectin could be a possible explanation of the drug's ineffectiveness [17]. Ketkar et al. suggested that further studies are needed to investigate in vivo effects of ivermectin on the ZIKV [17]. Study on male Sprague Dawley rats bone marrow cells in an in vivo study that received the combination of aged garlic extract (AGE) at doses of 300,600 and $1200 \mathrm{mg} \mathrm{kg}^{-1}$ with an ivermectin dose of $0.4 \mathrm{mg} \mathrm{kg}^{-1}$, as the minimum detectable toxic drug, showed a reduction in cytotoxic effects [18]. Perhaps it could be concluded that in the treatment of mice infected with ZIKV, higher doses of ivermectin could be given in combination with AGE, thus researchers would be able to better evaluate the antiviral effects of ivermectin at higher doses.

In a recently published in vitro study, researchers evaluated the effects of ivermectin on various cell lines infected with the ZIKV. The cells were infected with the ZIKV strain MR766 virus and in the $12 \mathrm{~h}$ post infection (HPI) exposed to a concentration of $20 \mu \mathrm{M}$ ivermectin. Researchers showed that nonstructural protein 5 (NS5), which is essential for viral RNA replication, requires both $\beta 1$ nuclear localization signal (NLS) and $\alpha / \beta$ NLS. Ivermectin also caused effective NS5 nuclear inhibition, so that after $7 \mathrm{~h}$ of treatment, a $60 \%$ reduction in NS5 levels was observed in the nucleus [19]. These findings are similar to some other studies [20, 21] that showed ivermectin inhibits the proliferation of dengue virus (DENV) by blocking NS5 interaction with IMP $\alpha / \beta$ transporter.

In a recently published in vivo and in vitro study, the effects of synthetic nanoparticle ivermectin (T-Fc-IVM-NP) were assessed on the ZIKV. In this study, human epithelial colorectal adenocarcinoma cells (Caco-2) and Balb/c Albino female mice were used. The results revealed that T-Fc-IVM-NP reduced NS1 protein expression, thus it could be a safe therapeutic against ZIKV [22].

The researchers found that the drug could cross the intestinal epithelial barrier after oral administration and reach a suitable concentration in the blood, while drug toxicity was reduced in epithelial cells and no liver toxicity was seen. Also, the study found a reduction in the expression of the NS1 protein in the ZIKV and concluded that the drug could be used as a safe treatment for the virus. Besides, in vitro evaluations showed that the drug did not cross the placental barrier and had temperature-dependent stability [22].

In an in vitro study [23], infected Vero cells by ZIKV with the multiplicity of infection (MOI) of one, 2 HPI were treated with ivermectin, and cell supernatant was analyzed quantitatively $22 \mathrm{~h}$ later using plaque assay and real-time quantitative RT-PCR (RT-q PCR), for virus production and proliferation, respectively.

Results revealed that ivermectin is a potent inhibitor of ZIKV with EC50 of 1-2 $\mu \mathrm{M}$ and ivermectin was not cytotoxic at the concentrations used. The researchers showed that ivermectin can dissociate IMP $\alpha / \beta 1$ heterodimer. Ivermectin was able to directly bind to IMP $\alpha$ armadillo connect repeat domain of IMP $\alpha$ and change structure/conformation, and this could be the basis for inhibited binding to IMP $\beta 1$. They concluded that ivermectin in a cell context could inhibit recognition by IMP $\alpha$ of NLS-containing proteins such as NS5. This study for the first time showed that ivermectin inhibits NLS recognition/nuclear targeting. The ability to inhibit IMP $\alpha-N L S$ binding in the cellular context was first demonstrated in this study using the bimolecular fluorescence complementation system. Ivermectin IMP $\alpha / \beta$ inhibitory mode of action has been confirmed previously [23, 24].

\section{Dengue virus, yellow fever virus (YFV), and West Nile virus (WNV)}

Kylie et al. in an in vitro study on infected human cervical adenocarcinoma cells (Hela) showed that ivermectin in high concentrations $(25-50 \mu \mathrm{M})$ has an inhibitory effect on the proliferation of DENV, a positive-sense, single-stranded RNA virus, the genus Flavivirus, the Flaviviridae family. It does this by inhibiting the transfer of viral proteins between the host cell cytoplasm and its nucleus, which is dependent on IMP $\alpha / \beta 1$. The researchers showed that ivermectin inhibited the nuclear aggregation of NS5 of DENV [21].

In another in vitro study of the flavivirus family, YFV, WNV, and DENV, the researchers found that ivermectin exerted its inhibitory effect by inhibiting the NS3 helicase domain and had no effect on the ATPase activity of helicase domains. In this study, ivermectin showed a stronger inhibitory effect on YFV and, to a lesser extent, inhibited the proliferation of WNV and DENV. The researchers confirmed that ivermectin exerts its effect against dsRNA unwinding activity by acting on the flavivirus helicase enzyme. The fact that ivermectin did not affect the helicaseassociated ATPase activity seems to be good because ATP is a key nucleotide in host cell metabolism. Ivermectin inhibited the flaviviral NS3 helicase, which mediates the RNA binding and unwinding mechanisms. The authors concluded that ivermectin acts as a highly specific inhibitor of intracellular viral RNA synthesis by targeting the activity of NS3 helicase in flaviviruses. In this study, the addition of drug before the first $14 \mathrm{~h}$ of entry of the virus into the cell showed a stronger antiviral effect against the YFV and this effect decreased significantly after the onset of intracellular RNA synthesis. It may be concluded that ivermectin could be effective in the early stages of infection and maybe a recommended drug for the prevention or treatment of early 
stages of viral infection, rather than advanced forms. Of course, confirmation of this statement requires further human studies and clinical trials [25].

In another study on four specific serotypes of DENV, results of treated infected Huh-7 cells with the ivermectin revealed its inhibitory effect on the IMP $\alpha / \beta$-mediated nuclear import. The authors cited the potential role of ivermectin as an antiviral drug in the treatment of DENV [20]. In an in vitro study of Vero cells infected with DENV stock: DENV2, New Guinea $\mathrm{C}$ strain, cells were exposed to $1-25 \mu \mathrm{M}$ of ivermectin $3 \mathrm{~h}$ before infection. A review of confocal laser scanning microscopy results revealed a significant NS5 protein in the cell cytoplasm. This finding suggests the transfer of NS5 via IMP $\alpha / \beta$, which was inhibited by ivermectin. Likewise, a significant reduction in nuclear accumulation of the green fluorescent protein aggregation (GFP)-NS5 was detected. Finally, the researchers showed a high and direct tendency of NS5 to IMP $\alpha / \beta$ [26]. In another study on human Huh-7 cells infected with DENV 1, DENV2, or DENV2 virus mouseadapted S221 strain, a fivefold reduction was seen in halfmaximal effective concentration $\left(\mathrm{EC}_{50}\right)$ of ivermectin while using liposomal systems as its nanocarriers, while the antiviral activity of the drug was significantly preserved [27].

In an in vitro study on DENV2 infected Vero cells with MOI of one, following 2 HPI the infected cells were treated with ivermectin, and cell supernatant was analyzed quantitatively $22 \mathrm{~h}$ later using plaque assay and RT-q PCR, for virus production and proliferation, respectively. Results revealed that ivermectin is a potent inhibitor of DENV2 (New Guinea C), with EC50 of $0.5 \mu \mathrm{M}$ and it was not cytotoxic at the concentrations used [23]. A phase III clinical trial in Thailand has been registered against DENV infection in which a single daily oral dose of ivermectin was declared to be safe, however, the final results [15] are not published yet.

\section{Hendra virus (HEV)}

In an in vitro study, researchers examined the effectiveness of ivermectin on HEV, a Henipavirus belonging to the Paramyxoviridae family and a negative-sense, singlestranded RNA virus. The main pathogenicity of this virus is partly due to its ability to inhibit the host type-one interferon response by producing the polycistronic $\mathrm{p}$ gene. In this study, researchers showed that HEV moves dynamically between the nucleus and the cytoplasm through the IMP $\alpha 1$. The study found that ivermectin inhibited $\mathrm{HEV}$ infection in mammalian cells and even reduced the virus by five times in a non-optimized single dose of 10 $\mu \mathrm{M}$, without drug cytotoxicity. The researchers concluded that ivermectin could be effective in treating HEV infection by inhibiting the transmission of the virus by IMP $\alpha 1 /$ $\beta 1$ [28].

\section{Newcastle virus}

In another in vitro and in vivo study, Azeem et al. studied the cytotoxicity of ivermectin and its potential antiviral effect on Newcastle virus, a negative-sense, single-stranded RNA virus from the paramyxoviridae family, on chick primary fibroblast cell line and 9-day-old chick embryo, respectively. In this study, ivermectin was tested at concentrations of $6.25,12.5,25,50,100$, and $200 \mu \mathrm{g} \mathrm{ml}^{-1}$, and results revealed that the drug at $100 \mu \mathrm{g} \mathrm{ml}^{-1}$ or above had cytotoxic effects. However, it was safe at concentrations of $50 \mu \mathrm{g} \mathrm{ml}^{-1}$ or less, drug cytotoxicity was not observed and a moderate to poor antiviral activity was noted [29].

\section{Venezuelan equine encephalitis virus (VEEV)}

Lundberg et al. evaluated the efficacy of ivermectin as an inhibitor of import $\alpha / \beta 1$, in cells infected with VEEV. It is an enveloped, non-segmented, single-stranded positivesense RNA virus from the Alphavirus genus, Togaviridae family. The drug reduced nuclear-associated capsid, virus titer, and cytopathic effects (CPE) caused by the virus. Although a limited reduction in virus replication was observed, this was not significant [30].

Based on previous study results, for the first time in an in vitro study, researchers investigated the effect of ivermectin on VEE $\mathrm{C}$ using in silico structure-based drug design. Results showed a reduction in viral replication, besides reduction in nuclear accumulation of capsid protein (Cap) in infected cells. In this study, which used VEEVC virus-infected Vero cells, the effect of ivermectin was examined along with two other drugs. In the concentration of $1 \mu \mathrm{M}$, ivermectin reduced the titer of the virus to a lesser extent than the other two drugs and researchers found that the antiviral mode of action of drugs was through the IMP $\alpha / \beta 1$ : C NLS interaction [31].

\section{Chikungunya virus (CHIKV), Semliki Forest virus (SFV), and Sindbis virus (SINV)}

In the study of baby hamster kidney cells or BHK-21 cell line infected with CHIKV which is an enveloped, positivesense, single-stranded RNA virus from the Alphavirus genus in Togaviridae family, ivermectin inhibited viral infection and eliminated the luciferase signal without significant drug toxicity $(P$ value $<0.001)$ [32].

Also, in both infected BHK-21 cell line and human hepatocellular Huh-7.5, luciferase was measured 16 and $18 \mathrm{~h}$ later, respectively, and the results showed a dramatic decrease in virus replication in human hepatocellular Huh7.5 cells. The results also showed that ivermectin is a potent inhibitor of both positive-strand and negative-strand RNA production. A strong decrease in virus protein expression 
was observed in infected cells, even at a high MOI. In this paper, ivermectin was very effective in inhibiting virus production compared with untreated specimens with $\sim 4 \operatorname{logs}$ as a potent antiviral inhibitor. Also, ivermectin, when used between $1.5 \mathrm{~h}$ before and at the time of infection, reduced the SFV titer by 2.3 logs in infected compared with noninfected cells but did not show a similar effect at later time points. Similar to the CHIKV infected cells, ivermectin gradually lost its effectiveness when added to later time points. However, when it was added before or at the same time of infection, it inhibited virus titers by 2 logs [32]. Again, as stated in previous studies [25], it can be concluded that ivermectin administration may be effective in the early stages of infection and could be recommended for the prevention or treatment of early stages of viral infection, rather than advanced forms. Of course, confirmation of this statement requires human studies and clinical trials.

In the same study [32], treatment with ivermectin in cells infected with other alphaviruses, including SFV and SINV, reduced virus production compared with noninfected cells. Ivermectin treatment also showed an inhibitory effect on the virus by reducing virus titers by 4 logs in YFV. All of these findings suggest a strong antiviral effect of ivermectin, as it has been able to effectively reduce viral RNA synthesis, viral RNA protein expression, and mature virion formation in infected cells with CHIKV. The authors concluded that ivermectin effect was due to its inhibitory property on two alphaviruses, including SFV and SINV, as well as its stronger inhibitory effect on YFV.

\section{Avian influenza A virus}

In an in vitro study using chicken hepatocellular carcinoma cells infected with Avian influenza A virus, which is a negative-sense, single-stranded, segmented RNA virus from the Orthomyxoviridae family, treatment with $10 \mu \mathrm{M}$ ivermectin completely prevented the nuclear transmission of different types of viral ribonucleoprotein complexes [33].

\section{Porcine Reproductive and Respiratory Syndrome virus (PRRSV)}

In another in vitro study of the antiviral effect of ivermectin in sub-cytotoxic doses on cultured porcine alveolar macrophage cells infected with PRRSV which is an enveloped, positive-stranded RNA virus from the Arteriviridae family, the cells were exposed to concentrations of $1-15 \mu \mathrm{M}$ ivermectin $1 \mathrm{~h}$ before infection as well as during the entire course of viral infection. The inhibitory effect of ivermectin on virus propagation was evident, and ivermectin significantly reduced the CPE caused by the virus and the expression of the virus gene in a dosedependent manner. At its highest dose, $15 \mu \mathrm{M}$, ivermectin caused a significant reduction in virus-infected cells, with a maximum inhibition of $95 \%$. In this study, the effective dose of the drug that inhibits $50 \%$ of viral infections $\left(\mathrm{ED}_{50}\right)$ was $6.7 \mu \mathrm{M}$, and the authors concluded that ivermectin effectively inhibited the proliferation of the PRRSV virus. The effect of ivermectin on reducing virus production decreased with time of infection, so that in a dose of $15 \mu \mathrm{M}$ of the drug in $1 \mathrm{~h}$ before infection, simultaneously with infection, and 1, 2, 4, and 12 HPI the virus production decreased from 80 to $42 \%$. In $24 \mathrm{HPI}$, no significant change in PRRSV propagation was observed. Based on these findings, the authors concluded that ivermectin, as an antiviral drug, is effective in initiating viral infection. Ivermectin caused a significant reduction in virus titer, indicating that the drug inhibited the optimal release of progeny virus from the natural host cell, but it did not inhibit the virus entry process. The strong inhibitory effect of ivermectin on the intracellular expression of PRRSV N protein, which resulted in a $90 \%$ reduction in its expression, indicates ivermectin's specific function against viral protein translation during virus replication. The amount of PRRSV $\mathrm{N}$ protein in the nucleus of infected cells treated with ivermectin did not change significantly, which indicates the inability of the drug to inhibit nuclear/nucleolar localization of $\mathrm{N}$. The drug also had an inhibitory effect on genomic RNA and subgenomic mRNA. The researchers acknowledged that ivermectin may impair the optimal synthesis of viral RNA by exerting its effect on the nonstructural protein 10 helicase, which has ATP-dependent helicase activity in the PRRSV virus, but more studies are needed to prove this hypothesis [34].

\section{Human immunodeficiency virus type 1}

HIV-1 is a single-stranded RNA virus, belonged to the genus Lentivirus within the family of Retroviridae. In an in vitro study, the researchers evaluated the effects of ivermectin as an inhibitor of HIV-1 nuclear protein transfer. The results showed that ivermectin reduced the NLScontaining protein binding by IMP $\alpha / \beta$ and inhibited this interaction at low concentrations (the half-maximal inhibitory concentration $\left.\left[\mathrm{IC}_{50}\right]: 4.8 \mu \mathrm{M}\right)$. Ivermectin significantly reduced nuclear accumulation GFP-IN by $P$ value $=0.003$ compared with the untreated control group and also significantly reduced $(P$ value $<0001)$ nuclear accumulation of GFP-tagged Op-T-NLS fusion protein. However, this study showed that ivermectin failed to control the nuclear accumulation of telomer repeat factor- 1 (GFP-TRF) as IMP $\beta 1$ is the only way to transfer it to the cell nucleus. Researchers concluded that ivermectin is not a specific inhibitor for IN -IMP $\alpha / \beta$ interaction, but it appears to be a specific inhibitor of cargos that are dependent on heterodimer to be 
transferred to the nucleus. The study concluded that ivermectin is a nuclear transport inhibitor via IMP $\alpha / \beta$, but does not affect the nuclear transfer via IMP $\beta 1$ alone, and also ivermectin completely inhibits nuclear import of the active integrase protein of HIV-1 as a critical component of the preintegration complex [35].

Kylie et al. in a study on infected human cervical adenocarcinoma cells (Hela) showed that ivermectin in high concentrations $(25-50 \mu \mathrm{M})$ has an inhibitory effect on the proliferation of HIV-1. It does this by inhibiting the transfer of viral proteins between the host cell cytoplasm and its nucleus, which is dependent on IMP $\alpha / \beta 1$. The researchers showed that ivermectin inhibited the nuclear aggregation of HIV-1 integrase [21].

\section{The antiviral effects of ivermectin on DNA viruses}

\section{Equine herpesvirus type 1 (EHV-1)}

A number of studies examined the antiviral effects of ivermectin on some DNA viruses. In an in vitro study of primary murine neurons infected with two different strains of EHV-1, which is a double-stranded DNA virus, ivermectin with different concentrations had no effect on strain Rac-H proliferation but reduced the proliferation of strain Jan-E. These findings suggest that different strains of EHV-1 use different receptors to enter the nucleus. Also, because ivermectin only inhibited the proliferation of strain Jan-E, further studies are needed to investigate the antiviral effect of ivermectin on this virus. The study's finding suggests the role of IMP $\alpha / \beta$ besides other receptors involved in nuclear import in the EHV-1 [36].

\section{Pseudorabies virus (PRV)}

$\mathrm{Lv}$ et al. examined the antiviral effect of ivermectin on an enveloped double-stranded DNA-based swine virus called PRV, which is a member of the alpha-herpesviridae subfamily [37]. The virus causes lifelong infection in pigs, and its DNA polymerase enzyme is made up of two subunits called UL30 and UL42 [38, 39].

The UL42 subunit is found to have IMP- $\alpha / \beta$-mediated bipartite NLS that transfers both subunits into the cell nucleus [39]. Examination of infected hamster kidney cells (BHK-21 cells) showed that ivermectin did not produce cytotoxic effects at concentrations $<3 \mu \mathrm{M}$. But with increasing the drug concentrations to $5 \mu \mathrm{M}$, the cells showed drug cytotoxic effects as a sharp decrease in cell activity. The CPE of viral infection were seen in untreated cells $24 \mathrm{HPI}$ and in cells treated with $0.5 \mu \mathrm{M}$ ivermectin in 48 HPI. In 72 HPI, mild CPE were seen in infected cells treated with 1.5 or $2.5 \mu \mathrm{M}$ ivermectin, indicating a delayed proliferation of the virus. In this study, ivermectin did not inhibit PRV adsorption in cells because the virus titers were the same in different groups. However, adding ivermectin after infection reduced the number of plaques and virus titers. Ivermectin inhibited the entry of DNA polymerase accessory subunit UL42 into the nucleus, so that with increasing the drug concentrations, less UL42 was observed in the nucleus by the western blot method. Although ivermectin inhibited the transfer of UL42 to the nucleus through the NLS, it did not reduce UL42 expression in the cytoplasm. In the virus-infected mice model, ivermectin significantly reduced viral loads in the brain and kidney of all animals, and this reduction was more significant in the kidneys, the main organ involved in ivermectin metabolism. In addition to declining virus titers in the organs of the animal, their clinical scores and mortality decreased as the drug concentration increased. Finally, the researchers concluded that ivermectin could be used as a potential antiviral drug against PRV [37].

\section{BK polyomavirus (BKPyV)}

As mentioned earlier, a study of Wagstaff et al. [21] showed that ivermectin was able to specifically inhibit the nuclear transfer pathway through IMP $\alpha / \beta$ [36]. Based on this mechanism, Bennet et al. investigated the effect of ivermectin on BKPyV, a non-enveloped small double-stranded DNA virus and a member of the Polyomaviridae family, in infected renal proximal tubule epithelial cells. A qualitative study using the reverse transcription-polymerase chain reaction method after treating infected cells with $10 \mu \mathrm{M}$ ivermectin, showed a decrease in the levels of the early protein large $\mathrm{T}$ Antigen mRNA, indicating a decrease in viral gene expression due to inhibition of nucleus entry. This inhibitory effect of ivermectin indicates that polyomavirus has access to the nucleus through active nuclear pore complex transfer [40].

\section{Porcine circovirus 2 (PCV2)}

The inhibitory effect of ivermectin on virus proliferation was investigated in PK-15 cells infected with PCV2, a circular single-strand DNA virus from the Circoviridae family. The results showed that ivermectin at concentrations of 50 or 100 $\mu \mathrm{g} \mathrm{ml}^{-1}$ did not have cytotoxic effects at 24 or $48 \mathrm{~h}$ after treatment, but at concentration of $200 \mu \mathrm{g} \mathrm{ml}^{-1}$ cell viability reduced significantly $(P$ value $\leq 0.05)$. Also in the first 24 HPI, ivermectin reduced the viral load by $41 \%$ and $28.2 \%$, at concentrations of 50 and $100 \mu \mathrm{g} \mathrm{ml}^{-1}$, respectively. However, in the $48 \mathrm{HPI}$, ivermectin reduced viral load by $28.8 \%$ and $15.7 \%$, respectively, at the same concentrations, indicating a decrease in drug efficacy in later time points [41], as was pointed out in previous studies on antiviral effects of ivermectin $[25,32]$.

Also in infected PK-15 cells, ivermectin reduced the expression of viral Cap, which has an NLS to enter the nucleus 
of an infected cell. Addition of ivermectin to the culture medium significantly reduced the number of virus-infected cells and following treatment, Cap caused by PCV2 infection was detected only in the cytoplasm and not in the nucleus [41].

Infected piglets treated with ivermectin showed a significant decrease $(P$ value $\leq 0.05)$ in viremia and viral loads in tissues. In the study of inguinal lymph nodes (ILNs) in infected piglets treated with ivermectin, the observed lesions were milder and there was a clear difference in the number of lymphocytes in the lymph nodes and the intensity of infiltration of the histiocytes [41].

Integrated optical density analysis of the PCV2 virus showed a significant decrease in viral signals in ILNs $(P$ value $\leq 0.05$ ) following treatment with ivermectin. Finally, the authors concluded that ivermectin inhibits the entry of Cap and the NLS of Cap in ILNs into the nucleus, which confirms the effect of drug on the NLS-mediated nuclear import pathway [41].

\section{Bovine herpesvirus 1 virus (BoHV-1)}

In another study on Madin-Darby bovine kidney cells infected with the BoHV-1, a large, enveloped and doublestranded DNA virus from the Herpesviridae family, ivermectin decreased UL42 nuclear transmission by inhibiting IMP $\alpha / \beta$-dependent nuclear transfer and reduced virus replication in a dose-dependent manner, indicating that UL4 2 was dependent on IMP $\alpha / \beta$ for nuclear transfer. 25 $\mu \mathrm{M}$ ivermectin reduced the virus titer by 4 logs and inhibited virion production by $\sim 44 \%$, but had no effect on cell viability in the studied doses. Also, ivermectin had no effect on the binding and entry of the virus into the host cell [42].

\section{Conclusion}

In this systematic review, we showed antiviral effects of ivermectin on a broad range of RNA and DNA viruses by reviewing all related evidences since 1970. This study presents the possibility that ivermectin could be a useful antiviral agent in several viruses including those with positive-sense single-stranded RNA, in similar fashion. Since significant effectiveness of ivermectin is seen in the early stages of infection in experimental studies, it is proposed that ivermectin administration may be effective in the early stages or prevention. Of course, confirmation of this statement requires human studies and clinical trials.

Ivermectin, owing to its antiviral activity, may play a pivotal role in several essential biological processes, therefore it could serve as a potential candidate in the treatment of different types of viruses including COVID-19. Clinical trials are necessary to appraise the effects of ivermectin on COVID19 in clinical setting and this warrants additional investigation for probable benefits in humans in the current and future pandemics. On April 10, 2020, FDA issued a statement concerning self-administration of ivermectin against COVID19 [43] referring to recently published in vitro study on this subject [15]. FDA highlighted that this type of in vitro study is usually used in the early stages of drug development. Moreover, further trials are needed to confirm the safety and efficacy of ivermectin for human use against COVID-19 to discover preventive or therapeutic window [43].

As noted, the activity of ivermectin in cell culture has not reproduced in mouse infection models against many of the viruses and has not been clinically proven either, in spite of ivermectin being available globally. This is likely related to the pharmacokinetics and therapeutic safety window for ivermectin. The blood levels of ivermectin at safe therapeutic doses are in the $20-80 \mathrm{ng} / \mathrm{ml}$ range [44], while the activity against SARS-CoV2 in cell culture is in the microgram range. Ivermectin is administered orally or topically. If safe formulations or analogs can be derived that can be administered to achieve therapeutic concentrations, ivermectin could be useful as a broad-spectrum antiviral agent.

\section{Compliance with ethical standards}

Conflict of interest The authors declare that they have no conflict of interest.

Publisher's note Springer Nature remains neutral with regard to jurisdictional claims in published maps and institutional affiliations.

\section{References}

1. Crump A, Ōmura S. Ivermectin, 'wonder drug' from Japan: the human use perspective. Proc Jpn Acad Ser B Phys Biol Sci. 2011;87:13-28. https://doi.org/10.2183/pjab.87.13.

2. Kircik LH, Del Rosso JQ, Layton AM, Schauber J. Over 25 years of clinical experience with ivermectin: an overview of safety for an increasing number of indications. J Drugs Dermatol. 2016;15:325-32.

3. Gonzalez Canga A, Sahagun Prieto AM, Diez Liebana MJ, Fernandez Martinez N, Sierra Vega M, Garcia Vieitez JJ. The pharmacokinetics and interactions of ivermectin in humans-a mini-review. AAPS J. 2008;10:42-6. https://doi.org/10.1208/s12248-007-9000-9.

4. Laing R, Gillan V, Devaney E. Ivermectin—old drug, new tricks? Trends Parasitol. 2017;33:463-72. https://doi.org/10.1016/j.pt. 2017.02.004.

5. Sohrabi C, Alsafi Z, O'Neill N, et al. World Health Organization declares global emergency: a review of the 2019 novel coronavirus (COVID-19). Int J Surg. 2020;76:71-6. https://doi.org/ 10.1016/j.ijsu.2020.02.034.

6. Lu H, Stratton CW, Tang Y-W. Outbreak of pneumonia of unknown etiology in Wuhan, China: the mystery and the miracle. J Med Virol. 2020;92:401-2. https://doi.org/10.1002/jmv.25678.

7. Ge X-Y, Li J-L, Yang X-L, et al. Isolation and characterization of a bat SARS-like coronavirus that uses the ACE2 receptor. Nature. 2013;503:535-8. https://doi.org/10.1038/nature12711.

8. Yang X-L, Hu B, Wang B, et al. Isolation and characterization of a novel bat coronavirus closely related to the direct progenitor of 
severe acute respiratory syndrome coronavirus. J Virol. 2015;90:3253-6. https://doi.org/10.1128/JVI.02582-15.

9. Lu C-C, Chen M-Y, Chang Y-L. Potential therapeutic agents against COVID-19: what we know so far. J Chin Med Assoc. 2020. https://doi.org/10.1097/JCMA.0000000000000318.

10. Ang L, Lee HW, Choi JY, Zhang J, Soo Lee M. Herbal medicine and pattern identification for treating COVID-19: a rapid review of guidelines. Integr Med Res. 2020;9:100407. https://doi.org/10. 1016/j.imr.2020.100407.

11. Gharebaghi R, Heidary F, Moradi M, Parvizi M. Metronidazole; a potential novel addition to the COVID-19 treatment regimen. Arch Academic Emerg Med. 2020;8:e40.

12. Baron SA, Devaux C, Colson P, Raoult D, Rolain J-M. Teicoplanin: an alternative drug for the treatment of COVID-19? Int J Antimicrob Agents. 2020:105944. https://doi.org/10.1016/j.ija ntimicag.2020.105944.

13. Gharebaghi R, Heidary F. COVID-19 and Iran: swimming with hands tied! Swiss Med Wkly. 2020;150:w20242. https://doi.org/ 10.4414/smw.2020.20242.

14. Reviglio VE, Osaba M, Reviglio V, Chiaradia P, Kuo IC. COVID-19 and ophthalmology: a new chapter in an old story. Med Hypothesis Disco Innov Ophthalmol. 2020;9:71-3.

15. Caly L, Druce JD, Catton MG, Jans DA, Wagstaff KM. The FDAapproved drug ivermectin inhibits the replication of SARS-CoV-2 in vitro. Antiviral Res. 2020:104787. https://doi.org/10.1016/j. antiviral.2020.104787.

16. Barrows NJ, Campos RK, Powell ST, et al. A screen of FDAapproved drugs for inhibitors of Zika virus infection. Cell Host Microbe. 2016;20:259-70. https://doi.org/10.1016/j.chom.2016. 07.004.

17. Ketkar H, Yang L, Wormser GP, Wang P. Lack of efficacy of ivermectin for prevention of a lethal Zika virus infection in a murine system. Diagn Microbiol Infect Dis. 2019;95:38-40. https://doi.org/10.1016/j.diagmicrobio.2019.03.012.

18. Khalil AM, Abu Samrah HM. In vivo combined treatment of rats with ivermectin and aged garlic extract attenuates ivermectininduced cytogenotoxicity in bone marrow cells. Res Vet Sci. 2018;120:94-100. https://doi.org/10.1016/j.rvsc.2018.09.005.

19. Ji W, Luo G. Zika virus NS5 nuclear accumulation is protective of protein degradation and is required for viral RNA replication. Virology. 2020;541:124-35. https://doi.org/10.1016/j.virol.2019. 10.010 .

20. Tay MYF, Fraser JE, Chan WKK, et al. Nuclear localization of dengue virus (DENV) 1-4 non-structural protein 5; protection against all 4 DENV serotypes by the inhibitor Ivermectin. Antivir Res. 2013;99:301-6. https://doi.org/10.1016/j.antiviral.2013.06. 002 .

21. Wagstaff KM, Sivakumaran H, Heaton SM, Harrich D, Jans DA. Ivermectin is a specific inhibitor of importin alpha/beta-mediated nuclear import able to inhibit replication of HIV-1 and dengue virus. Biochem J. 2012;443:851-6. https://doi.org/10.1042/ BJ20120150.

22. Surnar B, Kamran MZ, Shah AS, et al. Orally administrable therapeutic synthetic nanoparticle for Zika virus. ACS Nano. 2019;13:11034-48. https://doi.org/10.1021/acsnano.9b02807.

23. Yang SNY, Atkinson SC, Wang C, et al. The broad spectrum antiviral ivermectin targets the host nuclear transport importin alpha/beta1 heterodimer. Antivir Res. 2020;177:104760. https:// doi.org/10.1016/j.antiviral.2020.104760.

24. Kosyna FK, Nagel M, Kluxen L, Kraushaar K, Depping R. The importin alpha/beta-specific inhibitor Ivermectin affects HIFdependent hypoxia response pathways. Biol Chem. 2015;396:1357-67. https://doi.org/10.1515/hsz-2015-0171.

25. Mastrangelo E, Pezzullo M, De Burghgraeve T, et al. Ivermectin is a potent inhibitor of flavivirus replication specifically targeting
NS3 helicase activity: new prospects for an old drug. J Antimicrob Chemother. 2012;67:1884-94. https://doi.org/10.1093/jac/dks147.

26. Fraser JE, Rawlinson SM, Wang C, Jans DA, Wagstaff KM. Investigating dengue virus nonstructural protein 5 (NS5) nuclear import. Methods Mol Biol. 2014;1138:301-28. https://doi.org/10. 1007/978-1-4939-0348-1_19.

27. Croci R, Bottaro E, Chan KWK, et al. Liposomal systems as nanocarriers for the antiviral agent ivermectin. Int $\mathrm{J}$ Biomater. 2016;2016:8043983. https://doi.org/10.1155/2016/8043983.

28. Atkinson SC, Audsley MD, Lieu KG. et al. Recognition by host nuclear transport proteins drives disorder-to-order transition in Hendra virus V. Sci Rep. 2018;8:358. https://doi.org/10.1038/ s41598-017-18742-8.

29. Azeem S, Ashraf M, Rasheed MA, Anjum AA, Hameed R. Evaluation of cytotoxicity and antiviral activity of ivermectin against Newcastle disease virus. Pak J Pharm Sci. 2015;28:597-602.

30. Lundberg L, Pinkham C, Baer A, et al. Nuclear import and export inhibitors alter capsid protein distribution in mammalian cells and reduce Venezuelan equine encephalitis virus replication. Antivir Res. 2013;100:662-72. https://doi.org/10.1016/j.antiviral.2013. 10.004 .

31. Shechter S, Thomas DR, Lundberg L, et al. Novel inhibitors targeting Venezuelan equine encephalitis virus capsid protein identified using in silico structure-based-drug-design. Sci Rep. 2017;7:17705. https://doi.org/10.1038/s41598-017-17672-9.

32. Varghese FS, Kaukinen P, Glasker S, et al. Discovery of berberine, abamectin and ivermectin as antivirals against chikungunya and other alphaviruses. Antivir Res. 2016;126:117-24. https:// doi.org/10.1016/j.antiviral.2015.12.012.

33. Gotz V, Magar L, Dornfeld D, et al. Influenza A viruses escape from MxA restriction at the expense of efficient nuclear vRNP import. Sci Rep. 2016;6:23138. https://doi.org/10.1038/srep23138.

34. Lee YJ, Lee C. Ivermectin inhibits porcine reproductive and respiratory syndrome virus in cultured porcine alveolar macrophages. Arch Virol. 2016;161:257-68. https://doi.org/10.1007/ s00705-015-2653-2.

35. Wagstaff KM, Rawlinson SM, Hearps AC, Jans DA. An AlphaScreen(R)-based assay for high-throughput screening for specific inhibitors of nuclear import. J Biomol Screen. 2011;16:192-200. https://doi.org/10.1177/1087057110390360.

36. Slonska A, Cymerys J, Skwarska J, Golke A, Banbura MW. Influence of importin alpha/beta and exportin 1 on equine herpesvirus type $1(\mathrm{EHV}-1)$ replication in primary murine neurons. Pol J Vet Sci. 2013;16:749-51. https://doi.org/10.2478/pjvs-20130106.

37. Lv C, Liu W, Wang B, et al. Ivermectin inhibits DNA polymerase UL42 of pseudorabies virus entrance into the nucleus and proliferation of the virus in vitro and vivo. Antivir Res. 2018;159:55-62. https://doi.org/10.1016/j.antiviral.2018.09.010.

38. Berthomme H, Monahan SJ, Parris DS, Jacquemont B, Epstein AL. Cloning, sequencing, and functional characterization of the two subunits of the pseudorabies virus DNA polymerase holoenzyme: evidence for specificity of interaction. J Virol. 1995 May;69(5):2811-8. PubMed PMID: 7707503; PubMed Central PMCID: PMC188975

39. Wang Y-P, Du W-J, Huang L-P, et al. The pseudorabies virus DNA polymerase accessory subunit UL42 directs nuclear transport of the holoenzyme. Front Microbiol 2016;7:124. https://doi. org/10.3389/fmicb.2016.00124.

40. Bennett SM, Zhao L, Bosard C, Imperiale MJ. Role of a nuclear localization signal on the minor capsid proteins VP2 and VP3 in BKPyV nuclear entry. Virology. 2015;474:110-6. https://doi.org/ 10.1016/j.virol.2014.10.013.

41. Wang X, Lv C, Ji X, Wang B, Qiu L, Yang Z. Ivermectin treatment inhibits the replication of Porcine circovirus 2 (PCV2) in vitro and 
mitigates the impact of viral infection in piglets. Virus Res. 2019;263:80-6. https://doi.org/10.1016/j.virusres.2019.01.010.

42. Raza S, Shahin F, Zhai W, et al. Ivermectin inhibits bovine herpesvirus 1 DNA polymerase nuclear import and interferes with viral replication. Microorganisms. 2020;8. https://doi.org/10.3390/ microorganisms 8030409.

43. The FDA's Center for Veterinary Medicine, https://www.fda. gov/animal-veterinary/product-safety-information/fda-letter-sta keholders-do-not-use-ivermectin-intended-animals-treatmentcovid-19-humans. Accessed 5 May 2020.

44. Canga AG, Prieto AM, Liébana MJ, Martínez NF, Vega MS, Vieitez JJ. The pharmacokinetics and interactions of ivermectin in humans-a mini-review. AAPS J. 2008;10:42-6.

45. Crump A. Ivermectin: enigmatic multifaceted 'wonder' drug continues to surprise and exceed expectations. J Antibiot. 2017; 70:495-505. 